\title{
Research on the Method of Dynamic Emergency Rescue Vehicle Routing Based on Real-time Information
}

\author{
Tiejun Wang ${ }^{1,2}$ Yijun Li ${ }^{1}$ \\ ${ }^{1}$ School of Management, Harbin Institute of Technology, Harbin 150001, P. R. China \\ ${ }^{2}$ Heilongjiang Institute of Geomatics Engineering, Harbin 150001, P. R. China
}

\begin{abstract}
Vehicle routing problem is an important research topic in management science. Most traditional Vehicle routing studies are static models. Integrating the latest development of information technologies, such as "3S" geomatics technology and computer, communication and network modern high-techs, this study proposed an intelligent dynamic vehicle routing method and developed a dynamic emergency vehicle routing system based on real-time and historical information. Simulation results indicate that the efficiency of vehicle routing and the quality of emergency rescue services can be significantly improved with the proposed new method.
\end{abstract}

Keywords: Vehicle Routing, Emergency rescue, Real-time, Dynamic

\section{Introduction}

In all ages, human beings have been suffering from flood, earthquake and other natural disasters and malignant infectious diseases. So to speak, human civilization is developed on the basis of constantly responding to the challenges of all kinds of public emergencies. With the development of science and technology, the progress of the society, a variety of manmade accidents or technological disasters are increasing in recent years.
Under the present development level of science and technology, many types of emergencies, especially natural disasters are inevitable. But the damage caused by the emergencies can be significantly reduced or even eliminated through the application of modern science and technology for emergency rescue. Here into, the routing of emergency rescue vehicles, such as ambulances, fire engines, police cars, emergency logistic cars, etc is an important method.

The concept of Vehicle Routing Problem (VRP) was proposed by Dantzig and Ramserlal in $1959^{[1]}$, then it soon drew significant attention from the experts of the subjects of operational research, applied mathematics, combinational mathematics, graph theory and network analysis, logistics, computer application, etc. And it has been applied to the domains of express delivery, taxi service, logistics, production planning and emergency service and so on. VRP is a kind of academic research topic with broad practical value.

In the past few decades, most researches of this field are classical VRPs. In these problems, it is generally assumed that all the information, such as clients' location, the time of calling for services, the service volume, on-site service hours, running time of the vehicles are known before routing. And these information are independent of time, do not change with time running. In this assumption, the 
routings is relatively fixed. So these kinds of VRPs are called static VRPs.

However, there are a large number of uncertainties in the objective world, which reflected in the VRP, the clients' demands, the condition of the vehicles, the traffic jams, etc all can be uncertain. These kinds of uncertain information appear with the progress of time, then the routing of vehicles should be changed dynamically, the path of vehicles have been scheduled should be adjusted in good time. Especially in recent years, with the rapid social and economic development, different conditions all are in rapidly changing, and the dynamic realtime vehicle routing demands are constantly increasing. The static vehicle routing theories and methods are unable to meet the demands yet. The VRP is placed in the dynamic environment. On the other hand, this situation is much closer to the actual production and living, and as a result it begins to draw more and more attention.

The most significant characteristics of emergency response are the sudden happening and the pressing rescue, thus the dynamic real-time routing of emergency rescue vehicles is particularly important. In this research, three main technologies of geographic information science "3S" which are Geographic Information System (GIS), Global Positioning System (GPS) and Remote Sensing (RS), as well as other high-techs such as computer, communication, network are used as support, the dynamic routing of city emergency rescue vehicles is achieved based on real-time information and historical information, on the purpose of improving utilization efficiency of vehicles, and exploring the technical and methodological issues of the city emergency management.

\section{Background}

\subsection{Vehicle Routing Problem (VRP)}

The general definition of VRP is: To organize the appropriate routs for vehicles to go through a series of delivery and (or) pick-up spots orderly, and to satisfy certain constraints (such as material demanding and delivery quantity, delivery and pick-up time, vehicles capacity, running distance and time), and to achieve certain goals (for example, the shortest distance, least cost, minimal time, less vehicles).

Different factors are involved in VRP, and many models are generated. Some basic models are as follows:

(1) Capacitated Vehicle Routing Problem - CVRP

CVRP is the basic model of VRP. This model is with few constrains, commonly just subjected to capacity and driving time (or distance). It gets the longest research time and many results ${ }^{[2][3]}$. A large number of exact and heuristic algorithms are used to solve this problem, and the algorithms for solving other models are mostly derived from this.

(2) Vehicle Routing Problem with Time Windows - VRPTW

This model is the one which time window constrain is added to CVRP. It is close to the actual situation and the solving difficulty is increased. Many current researches focus on this model, such as literature [4][5], and a large number of intelligent optimization algorithms are proposed for this model. There are earliest service time and latest service time for each client as constraints. The Time window is divided into two categories: soft time windows (suffer penalty if could not meet the time constraint) and hard time windows (get infeasible solution if could not meet the time constraint).

(3) Vehicle Routing Problem with Pick-up and Delivery — VRPPD

Mainly two situations exist in this kind of problems, one is the client not only needs cargos but also return cargos. To simplify the problem, generally it is considered the returned cargos do not be ex- 
changed between clients but are shipped back to warehouse. The other situation needs to load goods from the pick-up clients and send them to the delivery clients. In this case, the order of the clients should be sequential. At the same time, the corresponding client to be paired and served by one vehicle. Article [6][7] studied this type of issues.

(4) Heterogeneous Fleet Vehicle Routing Problem - HFVRP

All vehicles are assumed to be the same type in general VRP research. But in real life, the vehicles may be of different types. Under this situation, Golden ${ }^{[8]}$ proposed HFVRP in 1984. In this kind of models, the number of each type of vehicle is subjected or not, and the costs of each vehicle type is different, the goal of optimizing is to minimize the overall costs of transportation or the number of vehicles. Article [9][10] are on such issues.

(5) Split Delivery Vehicle Routing Problem - SDVRP

Compared to general VRP, SDVRP allows a client to be served by two or more vehicles. Heuristic algorithms are always used for solving this problem. The articles [11][12] concerned this kind of problems.

\section{OVRP}

(6) Open Vehicle Routing problem -

The difference between OVRP and basic VRP is the vehicles are not required to return to the vehicle control center when they finish the tasks. Namely, the vehicle route is open not closed. Since 2000, some researchers began to conduct deep study on this issue. For instance, Brandao $^{[13]}$ used taboo search algorithm to solve OVRP. In addition, the article [14] [15] did research on this problem.

\subsection{Limitation of previous research}

Although a lot of researches have been done upon VRP, and the researches are rising currently. However, as a whole, for
VRP, especially for dynamic VRP, there are many important issues are waiting for further study. Present problems are mainly as follows:

(1) Most problems under study are static and with certainty. Dynamic and uncertainty problems are few, particularly realtime routing problems are very rare.

(2) How to integrate the existing hightech techniques (such as GPS, GIS, RS, communication, etc.) with classical VRP algorithms is not discussed in detail. It is worthy of studying how to get the benefits of rapid technological progress and how to use advanced techniques for vehicle routing services.

(3) The expanding researches of VRP are less. For example, the combination of VRP researches and practical problems of logistics, emergency rescue, and other domains are not deep enough.

This study uses " $3 \mathrm{~S}$ " geographic information technology, and computer, communications and network technologies etc in integration to achieve dynamic routing for emergency rescue vehicles based on real-time location information and traffic information, and various graphic and attribute information stored in GIS, including historical traffic information. It has important significance both in theory and practice.

\section{Methodology}

\subsection{Dynamic vehicle routing system}

(1) Composition and principle of system Dynamic real-time vehicles routing system consists of two parts: The master control part and the terminating units in C/S (Client / Server) pattern. The master control part is installed in the command center including GIS together with its database, the newest RS images, and GPS receiver as well as communication equipments. The terminals are equipped on each emergency rescue vehicles. The 
massive historical data and the RS images data can be stored in local machine; only the real-time information is interacted with master control part through the network. The GPS receiver and the essential communication equipments are needed too.

GIS is used to query historical data in database, including graph and attribute information to define the distance of the routes between two points that vehicles can go through. Simultaneously based on historical traffic information, as well as immediate feedback information from the vehicles, the current speed of vehicles can be determined; From these the running time of vehicles on each passable way can be calculated. Then the route of shortest time can be selected and the best routing scheme can be decided.

GPS is used to real-time position the vehicles; RS data provides the background images to assist analysis, judgment and decision-making; GSM/GPRS (Global System for Mobile Communications / General Packet Radio Service) and the network are used for the real-time communication and data transferring. System principles are shown in Figure 1.

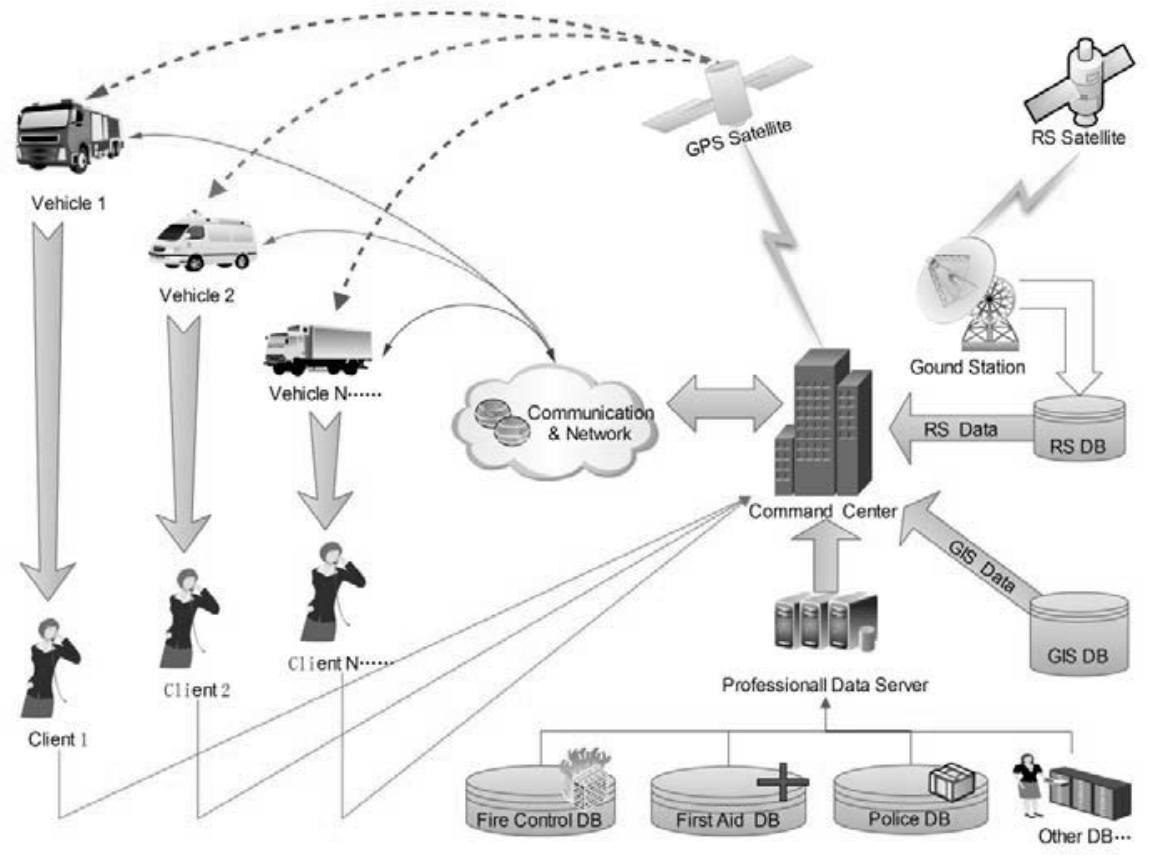

Fig. 1: System principle.

(2) Make initial routing scheme

Supposing some time is the beginning time, the command center queries the related information to locate the rescue scenes in GIS according to the emergency calls and then determines the positions of rescue spots. Then the positions will be indicated on the electronic map of vehicle routing system using GIS function. Simultaneously the map is transferred to the terminals on the vehicles through the network.

After positioning of the emergency scenes, together with the position and number of vehicles, as well as the historical information stored in GIS database, 
the routing scheme can be calculated using some algorithms, according to which the vehicles can be sent out. Simultaneously the command center monitors each vehicle's position in real-time manner by using GPS system, and demonstrates them on the electronic map.

Each vehicle also knows the location of itself by the GPS, and sees the targets will be served on the electronic map at any time. On the other hand, the vehicles should report the traffic information which is inconsistent with the historical information stored in the GIS database to the command center at any moment.

(3) Adjust routing scheme based on real-time information

When the following three types of situation happen: new emergency call appears; or any vehicle sent out to execute emergency rescue mission has completed the task and becomes the resource which can be dispatched, namely the vehicle situation changes; or the current reported traffic information differs with the historical information recorded in GIS database, the system will recalculate the optimal routing scheme according to the new information. The vehicles will drive to the new destinations immediately while receiving the adjusted routing scheme sent out from the command center.

\subsection{Dynamic vehicle routing model}

For emergency rescue, it is the VRP with hard time windows, and the earliest service time is 0 . Namely the vehicle must reach and serve the clients within the set time. Otherwise it will be regarded as the invalid solution. For example, the ambulances, the fire engines must arrive at the scene in 15 minutes after receiving the emergency call. In order to save the time and the cost, the vehicles do not return to the command center, but goes to the next client immediately after finishing serving one client, i.e. it is the open VRP. For simplifying the problem, the capacity and travel distance of vehicles will not be considered.

Supposing $i, j$ expresses the emergency points, the total number is $n$; There are $m$ vehicles $(\mathrm{m}<\mathrm{n}), x_{i j k}=1$ expresses the emergency rescue vehicle $k$ running from command center $(i=0)$ or client $i$ to client $j$ for service, otherwise $x_{i j k}=0 ; a_{i}, l_{i}$ respectively expresses the arriving and leaving time of client $i, s_{i j}$ expresses the service time at the location of client $i, t_{i j}$ expresses the running time of the vehicles from client $i$ to client $j ; d_{i j}$ expresses the driving distance between client $i$ and client $j$ (it is not the beeline distance but the route distance), $v_{i j}$ expresses the driving speed of the vehicles from client $i$ to client $j$.

Based on above conditions and the suppositions, the following model is established, the goal of which is to achieve the shortest total service time.

$$
\begin{aligned}
& \min \sum_{i=0}^{n} \sum_{j=0}^{n} \sum_{k=1}^{m} t_{i j} x_{j k} \\
& \text { s.t. } \sum_{i=0}^{n} \sum_{k=1}^{m} x_{i j k} \leq 1 \quad j=1, \ldots, \mathrm{n} \\
& \sum_{j=0}^{n} \sum_{k=1}^{m} x_{j k} \leq 1 \quad i=1, \ldots, \mathrm{n} \\
& \sum_{i=0}^{n} \sum_{k=1}^{m} x_{j k}-\sum_{i=0}^{n} \sum_{k=1}^{m} x_{j i k}=0 \quad j=1, \ldots, \mathrm{n} \\
& \sum_{i \in S} \sum_{j \in S} x_{j k} \leq|S|-1 \quad S \subseteq\{0, \ldots, \mathrm{n}\}, 2 \leq|S| \leq n-1, \forall k \quad \text { (5) } \\
& t_{i j}=d_{i j} / v_{i j} \quad \forall i, j \quad \text { (6) } \\
& \begin{array}{lll}
l_{i}=a_{i}+s_{i} & \forall i
\end{array} \\
& l_{i}+t_{i j} \leq a_{j} \quad \forall i, j \\
& x_{i k}=0 \text { 或 } 1 \quad \forall i, j, k \quad \text { (9) } \\
& i, j=0, \ldots, \mathrm{n} \\
& k=1, \ldots, \mathrm{m}
\end{aligned}
$$

In above model, formula (1) is the objective function, which requests the smallest overall service time. The constraint condition (2) and (3) express the vehicle reach and leave each client one time at most, i.e. each client will be served only by one vehicle at most one time; The constraint condition (4) controls flux conservation and guarantees the vehicle that reach one spot must leave there to a certainly; The constraint condition (5) is to 
eliminate the sub-loop; The constraint condition (6) expresses the relationship of the vehicle running time and the distance together with the speed; The constraint condition (7) expresses the relationship of three time variables: the time vehicles arrive at client $i$, the time vehicle leave client $i$, as well as the service time at client $i$; The constraint condition (8) also expresses the relations of three time variables: the time vehicle leave client $i$, the time vehicle arrive at client $j$, as well as the running time from client $i$ to client $j$. Formula (9) (11) restrict the variable value scope.

The above model is obviously a NPhard problem. If the client number is small, the exact algorithms can be used, like direct tree search algorithm, dynamic programming method and integer linear programming method and so on; If the client number is big, heuristic algorithms must be adopted, such as genetic algorithm, tabu search, simulated annealing algorithm, and ant colony optimization algorithm and so on.

\section{Simulation results}

Based on the dynamic vehicle routing model mentioned above, assuming the conditions of comparative crowded roads and busy system, simulating the prerequisite initial information for system calculation, such as the number of clients, the number of vehicles, historical GIS information, along with the real-time information of emergency needs, rescue vehicles, traffic, etc. Genetic algorithm is used for the calculation.

Meanwhile, using the same simulating data to do the calculation by static routing methods (supposing no real-time information available, all the information including the time and location of emergency call, the vehicle speed and the service time at clients' location, are known before routing scheme is determined, and will not change by time).

The results of static routing (SR) and dynamic routing (DR) are compared after stochastic 10 times calculation based on three targets: average service time for each client (AST, vehicle running time plus service time at client's location), the longest service time for one client (LST), and response rate of emergency calls (RREC). The comparison results are shown in Table 1.

\begin{tabular}{|c|c|c|c|c|c|c|}
\hline \multirow{2}{*}{ ID } & \multicolumn{2}{|c|}{$\begin{array}{c}\text { AST } \\
\text { (minute) }\end{array}$} & \multicolumn{2}{c|}{$\begin{array}{c}\text { LST } \\
\text { (minute) }\end{array}$} & \multicolumn{2}{c|}{$\begin{array}{c}\text { RREC } \\
\text { (\%) }\end{array}$} \\
\cline { 2 - 7 } & SR & DR & SR & DR & SR & DR \\
\hline 1 & 21 & 19 & 24 & 25 & 90 & 95 \\
\hline 2 & 26 & 23 & 28 & 29 & 90 & 90 \\
\hline 3 & 19 & 17 & 24 & 23 & 95 & 100 \\
\hline 4 & 23 & 20 & 26 & 27 & 90 & 95 \\
\hline 5 & 20 & 19 & 24 & 24 & 95 & 95 \\
\hline 6 & 18 & 16 & 21 & 22 & 100 & 100 \\
\hline 7 & 21 & 18 & 28 & 27 & 90 & 95 \\
\hline 8 & 24 & 22 & 29 & 30 & 85 & 85 \\
\hline 9 & 21 & 19 & 26 & 26 & 90 & 95 \\
\hline 10 & 23 & 21 & 25 & 27 & 85 & 90 \\
\hline
\end{tabular}

Table 1: Comparison results of static and dynamic routing.

\section{Conclusion}

This study applies the technologies of GIS, GPS, RS, GSM/GPRS etc into the designing of vehicle routing system, using geographic information as the carrier of other types of information, fully taking the advantages of current latest achievements in the development of related technologies.

In this study, the real-time information of vehicles and traffic, historical information in GIS are used for VRP decisions. The dynamic needs of clients and realtime uncertainty of emergency rescue vehicles and traffic are fully considered. It can improve the efficiency of emergency rescue vehicle routing, improve the utilization rate of emergency rescue vehicles 
and improve the quality of emergency rescue services. The design idea accords with the practicality and has strong academic investigation value and practical application significance.

The study results indicates that compared to static method, the dynamic routing method can evidently reduce the average service time for every client and increase the response rate of emergency calls, i.e. improve the working efficiency of emergency vehicles and the whole system. But the longest service time for one client is not increased distinctly, and even in the dynamic approach it may be slightly longer. This may be due to the reason that the system carries out the overall routing more reasonable under dynamic conditions, and reduces the average service time for every client, namely increases the number of clients served by each vehicle in unit time; At the same time it improves the response rate of emergency calls. That is to say, the dynamic routing system improves the utilization rate of emergency rescue vehicles. Certainly, in order to improve the overall efficiency and service quality, local service time had to be sacrificed - the server time of some clients will be longer.

Under current technical conditions, the computers are in superior performance, the network speed is fast, the mobile communications coverage rate is high. But if the system is huge, the situation of emergency calls expand instantaneously may occur, the calculating time for global optimizing of routing scheme could not be accepted. At this time, only local adjustment can be made. The result is not as good as the global one, but that is feasible. Of course, the bad situation of emergency calls could not be immediately responded, or no enough rescue vehicles to meet all the requests may appear. Then temporary increase of vehicles or other support should be needed.
Since the dynamic vehicle routing must deal with the information occurred at any moment in a real-time manner, it has higher request on the processing time. It is worthy of further research on the designing of clear structure, efficient algorithms. In addition, the validation of this article is based on simulated data, the model and method should be tested in practical application, further research is needed for improving and perfecting.

\section{References}

[1] Dantizig G., Ramser J. The truck dispatching problem $[\mathrm{J}]$. Management Science, 1959, 10 (6): 80-91.

[2] Laporte G., Mercure H., Nobert Y. An exact algorithm for the asymmetrical capacitated vehicle routing problem [J]. Networks, 1986, 16 (1): 33-46.

[3] Ralphs T. K. , Kopman L. , Pulleyblank W. R., Trotter L. E.. On the capacitated vehicle routing Problem [J]. Mathematical Programming, 2003, 94: 343-359.

[4] Duhamel C., Potvin J. Y., Rousseau J. M. A tabu search heuristic for the vehicle routing problem with backhauls and time windows $[\mathrm{J}]$. Transportation Science, 1997, 31 (1): 49-59.

[5] Marius M., Solomon M. Algorithms for vehicle routing and scheduling problems with time window constraints [J]. Operations Research, 1987, 35 (2): 76-78.

[6] Ahmad Alshamrania, Kamlesh Mathurb, and Ronald H. Ballou. Reverse logistics: simultaneous design of delivery routes and returns strategies [J]. Computers \& Operations Research, 2007, 34 (2): 595-619.

[7] Lu Quan, , Maged M. Dessouky. A new insertion-based construction heuristic for solving the Pickup and delivery Problem with time windows $[\mathrm{J}]$. 
European Journal of Operational Research, 2006, 175 (2): 672-687.

[8] Golden B., Assad A., Levy L., Gheysens F. The fleet zize and mix vehicle routing problem $[\mathrm{J}]$. Computers and Operations Research, 1984, 11 (1): 49-66.

[9] Eunjeong Choia, Dong-Wan Tchab. A column generation approach to the heterogeneous fleet vehicle routing Problem [J]. Computers \& Operations Research, 2007, 34 (7): 2080-2095.

[10]Tarantilis C. D., Kiranoudis C. K., Vassiliadis V. S. A threshold accepting metaheuristic for the heterogeneous fixed fleet vehicle routing problem [J]. European Journal of Operational Research, 2004, 152, (1): 148158.

[11]Mingzhou Jin, Kai Liu, Royee O. Bowden. A two-stage algorithm with valid inequalities for the split delivery vehicle routing Problem [J]. Interactional Journal of Production Economies, 2007, 105 (1): 228-242.

[12]Archetti C. Speranza M. Hertz A. A Tabu Search Algorithm for the Split Delivery Vehicle Routing Problem [J]. Transportation Science, 2006, 40 (1): 64-73.

[13]Jose Brandao. A tabu search algorithm for the open vehicle routing Problem [J]. European Journal of Operational Research, 2004, 157 (3): 552-564.

[14]Tarantilis C. D., Diakoulaki D. Kiranoudis C. K. Combination of geographical information system and efficient routing algorithms for real life distribution operations [J]. European Journal of Operational Research, 2004, 152 (2): 437-453.

[15]Repoussis P. P., Tarantilis C. D., Loannou $\mathrm{G}$. The open vehicle routing problem with time windows [J]. Journal of the Operational Research Society, 2007, 58 (13): 355-367. 